\title{
A simulação realística como ferramenta educacional para estudantes de medicina
}

\author{
Realistic simulation as an educacional tool for medical students \\ Carolina Felipe Soares Brandão', Carlos Fernando Collares'2, Heimar de Fatima Marin ${ }^{3}$
}

${ }^{1}$ Especialista em Administração Hospitalar pela Universidade de São Paulo (USP) e Mestre em Análises Clínicas pela Universidade de Santo Amaro (UNISA). Doutoranda do Departamento de Gestão e Informática em Saúde da Universidade Federal de São Paulo (UNIFESP), Coordenadora do Laboratório de Simulação Realística do Curso de Medicina da Universidade Cidade de São Paulo (UNICID), São Paulo, SP; ${ }^{2}$ Especialista em Medicina do Trabalho pela USP. Mestre em Psicologia pela Universidade Federal de Santa Catarina (UFSC). Doutor em Psicologia pela Universidade São Francisco (USF), Professor Assistente de Educação Médica da Universidade de Maastricht, Maastrich, Países Baixos; ${ }^{3}$ Professora Titular e Coordenadora do Programa de Pós-Graduação em Gestão e Informática em Saúde da UNIFESP, Mestre em Enfermagem e Doutora em Ciências Biológicas pela UNIFESP, Pós-doutora pela Havard Medical School, Livre Docente da USP, São Paulo, SP.

Apoio financeiro: Conselho Nacional de Desenvolvimento Científico e Tecnológico (CNPq) 301735/2009-3; Fogarty International Center and the National Library of Medicine, National Institutes of Health, Estados Unidos, Grant 5D43TW007015-08.

\section{RESUMO}

Objetivos: A simulação realística faz parte de uma nova possibilidade de ensino que engloba não somente as habilidades técnicas, mas o gerenciamento de crises, liderança, trabalho em equipe e raciocínio clínico que não reflitam prejuízos ao paciente real. O objetivo deste trabalho foi realizar revisão de literatura sobre a utilização da simulação realística, enfatizando a graduação médica e seus aspectos mais relevantes e atuais.

Fonte de dados: Foram consultadas as bases de dados PubMed e LILACS, aplicando-se os descritores patient simulation, students, medical e teaching/methods, considerando artigos de revisão publicados nos últimos três anos. Utilizando leitura flutuante dos resumos com a análise de conteúdo e dados registrados, foram encontrados 101 trabalhos de acordo com o propósito desta revisão.

Síntese dos dados: Os artigos relatam a importância e auxílio da tecnologia em agregar melhorias ao ensino médico. A segurança do paciente foi descrita como fator decisivo na implementação da simulação realística nas instituições de ensino mundiais. Diversas especialidades foram citadas nos artigos, além de diversos procedimentos específicos e aspectos de exame físico padronizado. Percebe-se interesse em estudar as possíveis formas de avaliar estudantes através dessa ferramenta. As habilidades em comunicação, liderança, tomada de decisão, trabalho em equipe e relacionamento médico/paciente aparecem nesta revisão como o maior benefício de informação aos estudantes.

Conclusões: Embora a simulação realística esteja em franco crescimento e valorizada como importante recurso na formação, novos estudos precisam ser realizados e divulgados para fornecer evidências e mensurações concretas e efetivas dessa ferramenta educacional.

DESCRITORES: SIMULAÇÃO DE PACIENTE; ESTUDANTES DE MEDICINA; ENSINO/métodos; SIMULAÇÃO REALíSTICA.

\section{ABSTRACT}

Aims: Realistic simulation is part of a new possibility of teaching that encompasses not only the technical skills, but crisis management, leadership, team work, and clinical reasoning that do not reflect actual losses to the real patient. To conduct a review of literature on the use of realistic simulation emphasizing the undergraduate medical education and its most relevant and current aspects.

Source of data: PubMed and LILACS databases were consulted, applying the medical subject headings patient simulation, students, medical and teaching/methods, considering articles published in the past three years. Using fluctuating reading of the abstracts with content analysis and recorded data and examination, 101 studies were found in accordance with the purpose of this review.

Summary of findings: Articles report the importance and support of technology in aggregating improvements to medical education. Patient safety was described as a decisive factor in the implementation of realistic simulation in institutions worldwide. Various specialties were mentioned among the articles in addition to several specific procedures and aspects of standardized physical examination. There is an interest in studying the possible ways to assess students through realistic simulation. Skills in communication, leadership, decision making, team work and relationship doctor/patient appear in this review as the greatest benefit of information to students.

Conclusions: Although realistic simulation is valued and have a rapid growth as an important resource in education, further studies need to be conducted and disseminated to provide concrete and effective evidences and measurements of this educational tool.

KEY WORDS: PATIENT SIMULATION; STUDENTS, MEDICAL; TEACHING/methods; REALISTIC SIMULATION. 


\section{INTRODUÇÃO}

A educação médica vêm sofrendo uma série de alterações, inclusões e novas perspectivas em relação ao ensino-aprendizagem. Tradicionalmente, a educação médica baseava-se em conhecimentos individuais, atitudes centradas em ensinamentos de professores, leituras de evidências científicas e práticas de procedimentos em pacientes reais. ${ }^{1} \mathrm{~A}$ fragmentação do conhecimento em especialidades e o aprendizado médico baseado em técnicas passivas, como por exemplo aulas teóricas e testes escritos, comprovadamente diminuem a retenção do conhecimento e aplicabilidade na prática. ${ }^{1,2}$

A simulação realística (SR) faz parte de uma nova possibilidade de ensino que engloba não somente as habilidades técnicas, mas o gerenciamento de crises, liderança, trabalho em equipe, raciocínio clínico em situações críticas ou que possam provocar prejuízos ao paciente real. Atualmente o termo simulação está empregado em diversas possibilidades de ensinoaprendizagem aos profissionais de saúde, o que promove muitas vezes certa confusão na aplicação destas distintas estratégias. As terminologias entre habilidades técnicas específicas ou part task trainer, uso de pacientes estandarizados e/ou padronizados, realidade virtual e simulação de alta fidelidade se misturam, porém todos eles contemplam diversas áreas de estudos na medicina, como emergências cardiológicas, trauma, pediatria, ginecologia e obstetrícia, cuidados intensivos, anestesia, habilidades atitudinais para a relação médico-paciente, entre várias outras (Tabela 1).

Há ainda outras estratégias, como a utilização de simuladores de baixa e média fidelidade, programas específicos de computadores e jogos virtuais, onde há também a possibilidade de simular uma situação específica. A escolha da estratégia deve estar ligada ao objetivo de aprendizagem, conteúdo prévio dos participantes, custos e capacitação docente adequada.

Dessa forma, a simulação hoje é vista como mais uma forma de aprendizagem, onde a retenção do conhecimento permanece por um tempo mais prolongado, além de ser uma estratégia mais agradável e prazerosa do que o ensino tradicional. ${ }^{1,3}$

Segundo Gaba, ${ }^{4}$ a simulação pode ser entendida como o processo educacional que replica cenários de cuidados ao paciente em um ambiente próximo a realidade, com o objetivo de analisar e refletir as ações realizadas de forma segura. Outra definição descrita por $\mathrm{Gaba}^{5}$ refere que simulação é uma técnica, e não exclusivamente uma tecnologia que tem por objetivo substituir ou amplificar uma experiência real com supervisão, mas que evoca substancialmente aspectos do mundo real em um ambiente interativo. Assim, a SR é uma ferramenta poderosa de aprendizado que pode ser aplicado em todos os níveis da educação médica, enfatizando a multidisciplinaridade em diversas situações clínicas. ${ }^{6}$

A ideia básica por trás da SR é promover a integração dos conhecimentos teóricos, habilidades técnicas e atitudinais, estimulando os estudantes a coordenarem todas as competências simultaneamente, facilitando assim a transferência do que foi aprendido para a solução de novos problemas. ${ }^{7}$ Especialmente em simulações de alta fidelidade e alta complexidade, faz-se necessária a reflexão do atendimento realizado pelos estudantes através de uma técnica chamada debriefing, onde o professor que acompanhou o atendimento terá uma postura de "facilitador" da discussão em grupo sobre os acertos e oportunidades de melhorias do cenário. A realização do debriefing pode ser auxiliada ou não com as imagens gravadas do atendimento, esclarecendo e revivendo de forma pontual momentos cruciais do atendimento oferecido ao paciente robô. Para as outras formas de simulação, a técnica de feedback é amplamente utilizada. Além disso,

Tabela 1. Definição das distintas e principais estratégias educacionais em simulação realística

\begin{tabular}{|ll}
\multicolumn{1}{c}{ Estratégia } & \multicolumn{1}{c}{ Definição Geral } \\
\hline Habilidades específicas - Part Task Trainer & $\begin{array}{l}\text { Manequins que permitem o treino de procedimentos específicos como otoscopia, } \\
\text { intubação orotraqueal, toque retal entre outros. Não necessariamente utilizam um } \\
\text { cenário contextualizando a situação. }\end{array}$ \\
\hline Paciente Estandarizado e/ou Padronizado - Standardized Patient & $\begin{array}{l}\text { Utilizam-se atores especializados em simulação, alunos treinados ou os próprios } \\
\text { pacientes. Muito utilizado para capacitação em habilidades comportamentais e } \\
\text { avaliação de estudantes. }\end{array}$ \\
\hline Simulação de alta fidelidade - High Fidelity Simulation & $\begin{array}{l}\text { Robôs que possuem respiração espontânea e são operados de forma a apresentar } \\
\text { hemodinâmica e procedimentos muito próximos ao real. Normalmente são utilizados } \\
\text { cenários trabalhados por uma equipe e que são discutidos em fase posterior } \\
\text { denominada debriefing. }\end{array}$ \\
\hline Realidade Virtual & $\begin{array}{l}\text { Bastante utilizado em simulações cirúrgicas onde há a necessidade de computação } \\
\text { gráfica para replicar um procedimento. }\end{array}$ \\
\hline Associação entre um manequim de habilidades técnicas e um paciente padronizado.
\end{tabular}


o emprego das técnicas de simulação permitem que se ofereçam as mesmas oportunidades de aprendizado, prática e treinamento para todos os estudantes de forma mais homogênea, sem depender de circunstâncias e do acaso, envolvidos no aprendizado baseado em situações reais. ${ }^{8}$ Deve-se ter em consideração que os estudantes de medicina não têm sempre acesso a uma ampla gama de experiências educacionais, o que acarreta uma menor oportunidade de observar e avaliar os pacientes com uma grande variedade de doenças, sinais físicos e sintomas. ${ }^{6,9,10}$ Neste caso a SR pode auxiliar efetivamente nas necessidades práticas dos estudantes em adquirir habilidades essenciais de forma que se consiga a proficiência do mesmo, promovendo a segurança e o bem-estar dos pacientes. , $^{6,11,12}$

A utilização da SR em universidades e escolas de medicina e outras áreas da saúde é extremamente recente no Brasil, especialmente na simulação de alta fidelidade, onde se exige um investimento financeiro alto em robôs e infraestrutura e é difícil a capacitação metodológica dos docentes. Nos Estados Unidos e Europa a SR já se encontra bem estabelecida e difundida como ferramenta indispensável ao ensino e educação continuada de profissionais de saúde, com o respaldo de efetivamente promover a segurança dos pacientes e participação prática dos alunos, gerando maior retenção do conhecimento discutido. Atualmente, destaca-se que a utilização de simuladores vem a ser considerada como um poderoso fator de redução de erros e de melhora do desempenho profissional. ${ }^{8,13}$ A SR proporciona melhor acesso à informação, ao conhecimento geral e às habilidades específicas, além de gerar altos índices de satisfação, não somente nos estudantes como nos docentes envolvidos no processo. ${ }^{6}$

Apesar dessa estratégia estar se difundindo rapidamente pelo mundo, enfatiza-se que não há o objetivo de substituir o processo de ensino-aprendizagem com o paciente real; a SR tem a intenção de proporcionar maior confiança e melhor preparo aos estudantes, não apenas tecnicamente mas no âmbito geral, garantindo a segurança aos pacientes e melhoria da qualidade no atendimento prestado.

O objetivo deste estudo foi realizar uma revisão de literatura integrativa e recente sobre o uso da SR na educação médica, priorizando o ensino de graduação e contemplando aspectos mais relevantes e atuais.

\section{MÉTODOS}

Para a busca de referências, foram consultadas as bases de dados PubMed e LILACS, aplicando-se à pesquisa os descritores (MeSH) patient simulation, students, medical e teaching/methods. Foram considerados artigos de revisão publicados de março de 2010 a março de 2013 e descritos na língua inglesa e portuguesa, sendo a pesquisa realizada no mês de julho de 2013. Para a organização das informações foi utilizada a leitura flutuante dos resumos dos trabalhos com a análise de conteúdo como verificação e com os dados devidamente registrados.

\section{RESULTADOS DA SELEÇÃO DOS ARTIGOS}

Foram encontrados 101 trabalhos, sendo que dois artigos foram excluídos por não contemplarem exclusivamente o período de graduação como fonte prioritária do estudo. Com os trabalhos divididos por tópicos, verificou-se que os temas mais comumente discutidos foram os relativos às habilidades em comunicação $e$ sobre avaliação através da SR; 82 manuscritos foram omitidos e 19 incluídos. Os 19 trabalhos foram agrupados em duas tabelas: artigos com foco na estratégia de simulação realística (Tabela 2) e artigos sobre comparação de estratégias em simulação (Tabela 3).

Tabela 2. Artigos publicados entre janeiro de 2013 e março de 2013 com foco na estratégia de simulação realística nos cursos de graduação em medicina e ciências da saúde.

\begin{tabular}{|c|c|c|c|}
\hline Ano & País & Autores & Tema \\
\hline 2013 & Alemanha & Schaufelberger $\mathrm{M}^{14}$ & O aumento da atratividade do primeiro atendimento na graduação médica. \\
\hline 2013 & Inglaterra & Peters $\mathrm{S}$ et al. ${ }^{15}$ & Mudança de comportamento entre estudantes de medicina em relação ao gerenciamento da obesidade. \\
\hline 2013 & Alemanha & Abendroth $\mathrm{M}$ et al. ${ }^{16}$ & Tomada de decisão: um piloto sobre e-learning. \\
\hline 2013 & Paquistão & Jabeen $\mathrm{D}^{17}$ & Uso de pacientes simulados para a avaliação de habilidades em comunicação em ginecologia e obstetrícia. \\
\hline 2013 & Holanda & de la Croix A et al. ${ }^{18}$ & O jogo simulado: uma análise de interação entre os estudantes e os pacientes simulados. \\
\hline 2013 & Austrália & Bearman $\mathrm{M}^{19}$ & Evitando o uso do tokenismo na educação de profissionais de saúde. \\
\hline 2013 & Holanda & Bouter $\mathrm{S}$ et al. ${ }^{20}$ & $\begin{array}{l}\text { Interpretação e validação do Nijmegen Evaluation of the Simulated Patient (NESP): avaliando a capacidade } \\
\text { dos pacientes simulados e feedback aos estudantes. }\end{array}$ \\
\hline 2013 & Estados Unidos & Colbert-Getz JM et al. ${ }^{21}$ & Como o gênero e ansiedade podem afetar a autoanálise dos estudantes em habilidades clínicas. \\
\hline 2013 & Estados Unidos & Hernandez C et al..$^{22}$ & $\begin{array}{l}\text { Avaliando a capacidade dos estudantes em detectar melanomas utilizando pacientes padronizados e } \\
\text { caracterizados (moulage). }\end{array}$ \\
\hline
\end{tabular}


Tabela 3. Artigos publicados sobre a comparação de estratégias em simulação

\begin{tabular}{|c|c|c|c|}
\hline Ano & Autores & Temas & Resultado \\
\hline 2010 & Bokken L, et al ${ }^{23}$. & $\begin{array}{l}\text { Instrução de pacientes reais e pacientes } \\
\text { simulados na graduação médica: um } \\
\text { experimento randomizado. }\end{array}$ & $\begin{array}{l}\text { Os alunos consideram autenticidade uma vantagem importante de pacientes } \\
\text { reais, porém o recrutamento difícil é uma desvantagem importante. Porém, } \\
\text { pacientes padronizados tem vantagens importantes comparadas com os } \\
\text { pacientes reais. Por exemplo, seus feedbacks. A escolha de contato com } \\
\text { pacientes reais para educação médica depende de fatores como a fase do } \\
\text { currículo e o propósito do conteúdo. }\end{array}$ \\
\hline 2010 & Bonnetain E, et al. ${ }^{24}$ & $\begin{array}{l}\text { Benefícios de sistemas de video no } \\
\text { aprendizado de procedimentos em } \\
\text { parada cardíaca. }\end{array}$ & $\begin{array}{l}\text { Simulações baseadas em telas de computador parecem ser efetivas em preparar } \\
\text { os alunos para o uso de simulação de alta fidelidade, as quais apresentam } \\
\text { simulações que são mais próximas a situações reais. }\end{array}$ \\
\hline 2010 & Wånggren $\mathrm{K}$, et al. ${ }^{25}$ & $\begin{array}{l}\text { Ensino do exame pélvico utilizando } \\
\text { pacientes padronizados: avaliação } \\
\text { de habilidades técnicas em estudo } \\
\text { controlado. }\end{array}$ & $\begin{array}{l}\text { Treinamento com pacientes padronizados são mais efetivos no ensino da técnica } \\
\text { de exame pélvico. Recomendamos que o uso de pacientes padronizados seja } \\
\text { considerado no treinamento dos estudantes. }\end{array}$ \\
\hline 2011 & Siebeck M, et al. ${ }^{26}$ & $\begin{array}{l}\text { Ensino do exame retal com simuladores: } \\
\text { efeito na retenção de conhecimento e } \\
\text { inibição. }\end{array}$ & $\begin{array}{l}\text { Ensino do exame retal com a ajuda de simuladores, que representam alta } \\
\text { fidelidade, podem ajudar alunos de graduação médica a superar a inibição } \\
\text { desse exame. } \\
\text { Simulação de pacientes padronizados é muito mais efetiva do que as que } \\
\text { utilizam manequins, que representam baixa fidelidade. Ambos os tipos de } \\
\text { simulação ajudam na aquisição de conhecimento. }\end{array}$ \\
\hline 2011 & Clever SL, et al. ${ }^{27}$ & $\begin{array}{l}\text { Percepção dos estudantes sobre o ensino } \\
\text { no ambulatório e pacientes simulados nas } \\
\text { habilidades em comunicação. }\end{array}$ & $\begin{array}{l}\text { O uso de pacientes/voluntários reais foi bem recebido por estudantes para o } \\
\text { ensino de técnicas de comunicação. } \\
\text { Expansão e estudos posteriores de participação desses voluntários irão } \\
\text { possibilitar melhor entendimento do seu papel principal em habilidades de } \\
\text { comunicação em relação aos pacientes padronizados. }\end{array}$ \\
\hline 2012 & Seago BL, et al. ${ }^{28}$ & $\begin{array}{l}\text { Treino de habilidades em exame pélvico } \\
\text { e genitais em manequins: a sequência } \\
\text { importa? }\end{array}$ & $\begin{array}{l}\text { Quando a simulação for usada para ensinar o exame pélvico para iniciantes, } \\
\text { pacientes padronizados parecem ser melhores, reduzindo a ansiedade e } \\
\text { melhorando o comprometimento com a simulação subsequente de habilidades } \\
\text { psicomotoras. }\end{array}$ \\
\hline 2012 & Schubart JR, et al. ${ }^{29}$ & $\begin{array}{l}\text { Uso de manequins de mama comparado } \\
\text { com pacientes padronizados no ensino } \\
\text { do exame clínico de mamas. }\end{array}$ & $\begin{array}{l}\text { Estudantes médicos que aprenderam a palpação da mama em manequim } \\
\text { tiveram uma performance tão boa ou melhor do que os que aprenderam } \\
\text { em pacientes padronizados, porém, uma análise de subgrupo revelou que o } \\
\text { benefício se limitou a alunos com menos experiência clínica. }\end{array}$ \\
\hline 2012 & Norman G, et al. ${ }^{30}$ & $\begin{array}{l}\text { O relacionamento entre a fidelidade } \\
\text { da simulação e a transmissão do apren- } \\
\text { dizado. }\end{array}$ & $\begin{array}{l}\text { Ambos aprendizados em alta ou baixa fidelidade resultaram em melhoras } \\
\text { consistentes em performance em comparação com grupos de controle sem } \\
\text { intervenção. Porém, quase todos os estudos não demonstram nenhuma } \\
\text { vantagem significativa da alta fidelidade em relação à baixa fidelidade, com } \\
\text { médias de diferenças variando entre } 1 \% \text { e } 2 \% \text {. }\end{array}$ \\
\hline 2012 & Parekh A, et al. ${ }^{31}$ & $\begin{array}{l}\text { Como devemos ensinar os graduandos } \\
\text { em cenários simulados? }\end{array}$ & $\begin{array}{l}\text { Os dois estilos diferentes de simulação complementam um ao outro e } \\
\text { juntos oferecem uma experiência de aprendizado mais rica para os alunos. } \\
\text { Nós sugerimos que os estudantes considerem estilos diferentes de ensino de } \\
\text { simulação para maximizar a retenção de conhecimento. }\end{array}$ \\
\hline 2012 & Elley CR, et al. ${ }^{32}$ & $\begin{array}{l}\text { Efetividade da simulação clínica no } \\
\text { ensino da prática clínica: um controle } \\
\text { randomizado. }\end{array}$ & $\begin{array}{l}\text { Simulações clínicas podem auxiliar no desenvolvimento de habilidades de } \\
\text { comunicação dentro de práticas gerais de consulta e podem também ser } \\
\text { utilizado para complementar a comunicação baseada na comunidade com } \\
\text { pacientes reais. } \\
\text { Mesmo assim, confiança no gerenciamento de condições comuns e proce- } \\
\text { dimentos melhoram mais com pacientes reais }\end{array}$ \\
\hline
\end{tabular}

\section{CONTEÚDO DA REVISÃO}

\section{Implementação da simulação realística como estratégia educacional}

De modo geral, todos os artigos relatam a importância e auxílio dos recursos tecnológicos em agregar melhorias à SR como estratégia educacional no ensino médico e das demais disciplinas da saúde. A segurança do paciente também foi descrita como fator decisivo na implementação dessa metodologia nas diferentes instituições de ensino mundiais. Diversas especialidades foram citadas nos artigos, tais como cardiologia, ginecologia e obstetrícia, dermatologia e psiquiatria (habilidades em comunicação em geral), além de diversos procedimentos específicos, como toque retal e exame das mamas, entre outros aspectos de exame físico padronizado. Percebe-se um crescimento no interesse de estudar as possíveis formas de avaliar estudantes através dessa ferramenta, de forma objetiva e que demonstre credibilidade e reprodutibilidade, entre as diversas escolas que integraram a SR nos seus currículos, assim como estudos que realizaram comparações entre as possíveis estratégias de simulação (Tabela 3). As habilidades em 
comunicação, liderança, tomada de decisão e trabalho em equipe, assim como relacionamento entre médico/ profissional de saúde e paciente, aparecem nesta revisão como o maior benefício de informação aos estudantes inserido na metodologia de SR.

\section{Simulação realística no ensino de graduação}

Embora seja relativamente nova a utilização da SR em especial na graduação, em especial no Brasil, percebe-se que esta estratégia vêm crescendo de forma rápida com a adoção de novas tecnologias, entretanto a maioria dos estudos em simulação estão voltados para o treinamento de habilidades técnicas específicas ou comportamentais com residentes ou profissionais graduados, o que dificulta a inserção curricular plena dessa metodologia. Outro quesito relevante é a escassez de estudos voltados para a simulação de alta fidelidade durante a graduação, proporcionando maior segurança as instituições de ensino em investir nestes equipamentos, que são onerosos e se tornam obsoletos com certa rapidez. No Brasil há dificuldade não somente pelo quesito financeiro, uma vez que esta estratégia é onerosa às instituições, mas também a dificuldade de capacitação docente, que é fundamental para a condução de qualquer metodologia ativa.

Muitos dos artigos de revisão relatam a percepção dos participantes/estudantes desta estratégia educacional, porém esta análise do estudante ou até mesmo do profissional formado não deve ser utilizada como uma ferramenta para mensurar com precisão as competências adquiridas ou até mesmo sua retenção de conhecimento isoladamente, portanto fazem-se necessários mais trabalhos sobre esse recurso educacional, especialmente na graduação, onde há uma abordagem menor de estudos em comparação à educação continuada e ao treinamento de residentes. Mesmo neste contexto, segundo artigo de Bokken et al., ${ }^{33}$ os estudantes, ao compararem o paciente real com o simulado, acreditam que as duas metodologias são indispensáveis à construção do conhecimento.

A educação baseada em simulação aos graduandos de medicina contempla sem dúvida inúmeros quesitos fundamentais na construção do conhecimento, que muitas vezes não são abordados ou vivenciados durante o curso, proporcionando uma experiência ímpar e ativa de reflexão sobre os conteúdos abordados.

A associação de metodologias ativas com a evolução acelerada da tecnologia e da informática em saúde busca a cada dia melhorar o conteúdo de habilidades técnicas e não técnicas, além do raciocínio clínico de alunos de medicina, replicando de forma segura e muito próxima da real situações críticas que poderão ser enfrentadas na vida real destes profissionais. Entretanto a sua evidência de efetividade especialmente durante a graduação ainda é fraca na literatura e maiores estudos são necessários para se determinar sua real efetividade. ${ }^{34}$

\section{CONSIDERAÇÕES FINAIS}

O desenvolvimento de estratégias ativas de ensino aprendizagem que utilizam a SR proporcionam condições de integração dos conhecimentos aos estudantes e se propõem a formar profissionais mais críticos, reflexivos e preparados para a atuação real. Esta revisão de literatura integrativa e recente sobre o uso da SR na educação médica de graduação permitiu verificar que embora valorizada e entendida como importante recurso na formação profissional, novos estudos precisam ser realizados e divulgados para fornecer evidências concretas e efetivas sobre sua real retenção de conhecimento e desta forma facilitar e justificar a implementação dessas estratégias em cursos de graduação em medicina e outras ciências da saúde.

\section{AGRADECIMENTOS}

A autora Heimar de Fatima Marin expressa agradecimentos ao apoio fornecido pelo CNPq (301735/2009-3) e pelo Grant 5D43TW007015-08, Fogarty International Center and National Library of Medicine, National Institutes of Health, Estados Unidos.

\section{REFERÊNCIAS}

1. Flato UA, Guimarães HP. Educação baseada em simulação em medicina de urgência e emergência: a arte imita a vida. Rev Bras Clin Med. 2011;9(5):360-4.

2. Khan K, Pattison T, Sherwood M. Simulation in medical education. Med Teach. 2011;33(1):1-3.

3. Brim NM, Venkatan SK, Gordon JA, Alexander EK. Long-term educational impact of a simulator curriculum on medical student education in an internal medicine clerkship. Simul Healthc. 2010;5(2):75-81. 
4. Gaba DM. Do as we say, not as you do: using simulation to investigate clinical behavior in action. Simul Healthc. 2009;4(2):67-9.

5. Gaba DM. The future vision of simulation in healthcare. Simul Healthc. 2007;2(2):126-35.

6. Ypinazar VA, Margolis SA. Clinical simulators: applications and implications for rural medical education. Rural Remote Health. 2006;6(2):527.

7. van Merrienboer J, Kirschner P. Ten steps to complex learning: a systematic approach to four component instructional design. Mahwah (NJ): Lawrence Erlbaum Associates; 2007.

8. Troncon LE, Maffei CM. A incorporação de recursos de simulação no curso de graduação em medicina da Faculdade de Medicina de Ribeirão Preto - USP. Medicina (Ribeirão Preto). 2007;40(2):153-61.

9. Stumberg JP, Reid A, Khadra MH. Community based medical education in a rural area: a new direction in undergraduated training. Aust J Rural Health. 2001;9(Suppl 1):S14-8.

10. O'Sullivan M, Martin J, Murray E. Students'perceptions of the relative advantages and disadvantages of community-based and hospitalbased teaching: a qualitative study. Med Educ. 2000;34(8):648-55.

11. Flanagan B, Nestel D, Joseph M. Making patient safety the focus: crisis resource management in the undergraduated curriculum. Med Educ. 2004;38(1):56-66.

12. Bradley P, Postlethwaite K. Setting up a clinical skills learning facility. Med Educ. 2003:37(Suppl 1):6-13.

13. Ziv A, Ben-David S, Ziv M. Simulation based medical education: an opportunity to learn from errors. Med Teach. 2005;27(3):193-9.

14. Schaufelberger M. [Increase of attractiveness of primary care during undergraduate medical education]. Praxis (Bern 1994). 2013;102(6): 341-4.

15. Peters S, Bird L, Ashraf H, Ahmed S, McNamee P, Ng C, Hart J. Medical undergraduates' use of behaviour change talk: the example of facilitating weight management. BMC Med Educ. 2013;13:7.

16. Abendroth M, Harendza S, Riemer M. Clinical decision making: a pilote-learning study. Clin Teach. 2013;10(1):51-5.

17. Jabeen D. Use of simulated patients for assessment of communication skills in undergraduate medical education in obstetrics and gynaecology. J Coll Physicians Surg Pak. 2013;23(1):16-9.

18. de la Croix A, Skelton J. The simulation game: an analysis of interactions between students and simulated patients. Med Educ. 2013;47(1): 49-58.

19. Bearman M, Ajjawi R. Avoiding tokenism in health professional education. Med Educ. 2013;47(1):9-11.

20. Bouter S, van Weel-Baumgarten E, Bolhuis S. Construction and validation of the Nijmegen Evaluation of the Simulated Patient (NESP): assessing simulated patients' ability to role-play and provide feedback to students. Acad Med. 2013;88(2):253-9.

21. Colbert-Getz JM, Fleishman C, Jung J, Shilkofski N. How do gender and anxiety affect students' self-assessment and actual performance on a high-stakes clinical skills examination? Acad Med. 2013;88(1):44-8.

22. Hernandez C, Mermelstein R, Robinson JK, Yudkowsky R. Assessing students' ability to detect melanomas using standardized patients and moulage. J Am Acad Dermatol. 2013;68(3):e83-8.

23. Bokken L, Rethans JJ, Jöbsis Q, Duvivier R, Scherpbier A, van der Vleuten C. Instructiveness of real patients and simulated patients in undergraduate medical education: a randomized experiment. Acad Med. 2010;85(1):148-54.

24. Bonnetain E, Boucheix JM, Hamet M, Freysz M. Benefits of computer screen-based simulation in learning cardiac arrest procedures. Med Educ. 2010;44(7):716-22.

25. Wånggren K, Fianu Jonassen A, Andersson S, Pettersson G, Gemzell-Danielsson K.Teaching pelvic examination technique using professional patients: a controlled study evaluating students' skills. Acta Obstet Gynecol Scand. 2010;89(10):1298-303.

26. Siebeck M, Schwald B, Frey C, Röding S, Stegmann K, Fischer F. Teaching the rectal examination with simulations: effects on knowledge acquisition and inhibition. Med Educ. 2011;45(10):1025-31.

27. Clever SL, Dudas RA, Solomon BS, Yeh HC, Levine D, Bertram A, et al. Medical student and faculty perceptions of volunteer outpatients versus simulated patients in communication skills training. Acad Med. 2011;86(11):1437-42.

28. Seago BL, Ketchum JM, Willett RM. Pelvic examination skills training with genital teaching associates and a pelvic simulator: does sequence matter? Simul Healthc. 2012;7(2):95-101.

29. Schubart JR, Erdahl L, Smith JS Jr, Purichia H, Kauffman GL, Kass RB. Use of breast simulators compared with standardized patients in teaching the clinical breast examination to medical students. J Surg Educ. 2012;69(3):416-22.

30. Norman G, Dore K, Grierson L. The minimal relationship between simulation fidelity and transfer of learning. Med Educ. 2012 Jul;46(7):636-47.

31. Parekh A, Thorpe T. How should we teach undergraduates in simulation scenarios? Clin Teach. 2012;9(5):280-4.

32. Elley CR, Clinick T, Wong C, Arroll B, Kennelly J, Doerr H, et al. Effectiveness of simulated clinical teaching in general practice: randomised controlled trial. J Prim Health Care. 2012;4(4):281-7.

33. Bokken L, Rethans JJ, van Heurn L, Duvivier R, Scherpbier A, van der Vleuten C. Students' views on the use of real patients and simulated patients in undergraduate medical education. Acad Med. 2009;84(7):958-63.

34. Weller JM. Simulation in undergraduate medical education: bridging the gap between theory and practice. Med Educ. 2004;38(1):32-8. 